Int. J. Dev. Biol. 48: 739-742 (2004)

doi: $10.1387 / \mathrm{ijdb} .041918$ to

\title{
From embryonic induction to cell lineages: revisiting old problems for modern study
}

\author{
TOKINDO S. OKADA* \\ JT Biohistory Research Hall, Takatsuki, Osaka, Japan
}

\begin{abstract}
A history of embryological studies of lens development and regeneration is sketched, paying special attention to the contribution of these studies to the conceptual aspect of embryology and later developmental biology. Emphasis is made on the fact that the interaction of different tissues during development, namely embryonic induction, was first discovered during studies of the lens and that the degree of the dependence of lens development on the inductive effect of the eye-cup varies among different species. Studies along the line of comparativeexperimental embryology at the species level should be informative, in particular in combination with the recent trend of evo-devo studies. The processes of lens regeneration and in vitro transdifferentiation indicate the existence of multiple cell lineages with the potential of lens development in one animal. The occurrence of Wolffian lens regeneration in nature can be reconsidered from the new "eco-devo" viewpoint.
\end{abstract}

KEY WORDS: lens, eye-cup, transdifferentiation, Wolffian regeneration, evo-devo, eco-devo

\section{A choice of developmental mechanisms: dependent or independent of the eye-cup?}

From the beginning of experimental embryology, tracing back to the turn of the 20th century, studies of lens development have attracted the keen interest of embryologists and cultivated some of the most central problems of development. These studies were not only fruitful in providing important information, but also led to new concepts of tissue interactions in development. Micro-surgical techniques applied to living embryos opened up completely new experimental approaches for the analysis of development (see Okada, 2000). Many important problems were raised in that era, but have long since escaped the attention of modern researchers.

It is well established that interactions between different parts of embryos - inductive interactions - constitute one of the fundamental principles of animal development. This was first discovered through studies of lens formation in amphibian embryos (Spemann, 1901; see Saha etal., 1991 and Sander \& Faessler, 2001). The discovery was made possible by the use of a microsurgical technique which was first applied to embryos, namely removal of the eye-cup underlying the surface ectoderm. This traditional technique is still in use, demonstrating the unparalleled potential of experimental embryology.

Nowadays, however, we tend to be ignorant about the fact that the inductive effect of the eye-cup to elicit lens development is not at all a universal principle even among amphibians. Immediately after the first announcement of eye cup-dependent lens formation in Rana fusca (Spemann, 1901; see Fig. 1), H. Spemann himself carried out the same experiment using another frog species $R$. esculenta and observed a completely different outcome, namely occurrence of lens development even after removal of the eyecup anlage. This sharp contrast must have been a great puzzle and surprise to Spemann.

To explain such a great discrepancy, Hans Spemann introduced a kind of semi-philosophical concept (or a metaphor) which he called double assurance (Doppelte Sicherung, in the original German). His discussions leading to the concept had a somewhat philosophical or even teleological flavor, and were not generally appreciated, despite the clear and reliable experimental evidence (see Spemann, 1938). The concept of double assurance can be summarized in the following way: since developmental processes are of critical importance for life, all organisms prepare multiple potential ways of development to guarantee its successful accomplishment.

One species chooses a mechanism highly dependent on the effect of the eye-cup to initiate lens development, whereas another employs a mechanism independent of the process. Here is a problem of self-conflicting comparative-experimental embryology. Comparative embryology was a leading field of embryology and provided an important source of information for the foundation of evolutionary study in the last century. On the other hand, experimental embryology had at its historical root an idea principally

\footnotetext{
*Address correspondence to: Prof. Tokindo S. Okada. 22, Minamishiba-cho, Shimogamo, Sakyo-ku, Kyoto 606-0841, Japan. Fax: +81-75-781-1105.

e-mail: eiko788@yahoo.co.jp
} 


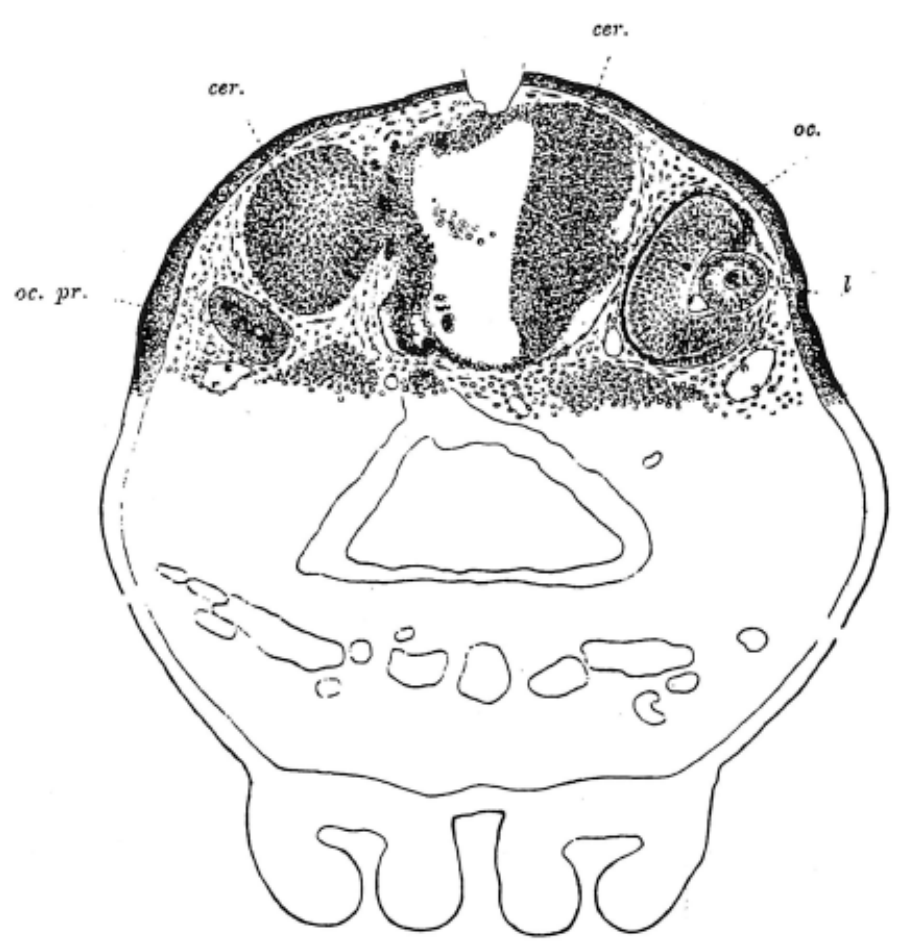

Fig. 1. A section of the head of a Rana fusca larva after removal of anlage of optic vesicle from the left side of the neurula. No lens formation was seen on the operated side, while formation of a normal lens (I) was observed on the control side (from Spemann, 1901, see also Okada, 2000).

incompatible with comparative embryology: it did not aim to understand the evolution of animal species. Experimental embryology pursued experimental analysis to produce a universal theory of development, disregarding evolutional diversity.

Thus, the standpoint of comparative-experimental embryology has been slow to gain popularity. However, it has blossomed today under the guise of Evo-Devo, especially at the molecular level. In the past we avoided questions such as those concerning the evolutionary origin of induction, or the evolutionary advantage of induction mechanisms. How could the inductive mechanism dependent on the eye-cup have become more prevalent than that which was independent of the eye-cup during the process of evolution, at least in the normal and healthy development of organisms? This question was uniquely tackled by D. P. Filatov, in Russia. Most of his scientific reports were written in Russian, but fortunately enough Dettlaff and Vassetzky (1997) introduced his unique contribution in concise English-written literature, enabling us non-Russian scientists to obtain access to his work. The favorite animals of D. P. Filatov, besides frogs, were pike and sturgeons, and he studied ears and lenses (Fig. 2).

According to Dettlaff and Vassetzky (1997), D. P. Filatov referred to his comparative approach as "a method of putting the static into motion and thus revealing its hidden properties in development". In other words, only a fraction of the developmental potentials are explicit by observation of an animal, while comparison of various animal species is revealing of full potentials. This notion gains support when the process of ontogenic lens development is compared with that of lens regeneration, which will be discussed later.
Recently, we ourselves compared dependence of lens development on inductive interaction with the eye-cup using two amphibians: Xenopus laevis and Cynops (Triturus) pyrrhogaster (Mizuno et al., 1998). The results indicated a clear difference when the eye-cup was removed in the mid-neurula stage. Neither morphological lens development nor expression of $\alpha \mathrm{A}-, \beta \mathrm{B}$ - and $\gamma$ crystallin genes occurred in Cynops, but these took place at least partly in Xenopus. Thus, major differences exist in developmental mechanisms for lens formation among different species even within amphibian species. Each animal species appears to rigidly choose only one of the mechanisms, with no statistical variations from one mechanism to another.

Was such a choice made in the process of evolution? In my view, the difference in the dependence on eye-cup interaction of lens development is not particularly relevant to global evolutionary alterations involving a higher level of systematic zoology, because the difference in choice of a mechanism for lens development occurs at the species level, even in the same genus. This problem was challenged in a series of studies carried out by R. Grainger's group, suggesting that differences in the timing of action of the eye-cup contribute to this difference (see Saha et al., 1989; Servetnick et al., 1996). It will be interesting to discover the basis of their similarities and differences at the molecular level.

Looking back to the middle of the last century, an important indication that one species possesses multiple parallel mechanisms for lens formation, which may be manifested under different environmental cues, e.g. temperature, was provided by a series of experiments carried out by scientists of the old Dutch school, including M. W. Woerdeman and his colleagues (see Twitty, 1955). They used the same $R$. esculenta as H. Spemann, but failed to observe lens formation after the removal of eye-cup in embryos previously reared at $25^{\circ} \mathrm{C}$. However, using embryos reared at $10^{\circ} \mathrm{C}$ they did reproduce $\mathrm{H}$. Spemann's original finding of eye-cupindependent lens formation. The Dutch scientists interpreted this difference as further advancement of internal chemical changes in the lens-forming ectoderm at $10^{\circ} \mathrm{C}$ in comparison to $25^{\circ} \mathrm{C}$. This is an interesting and somewhat rare example of an environmental influence upon a developmental process. It would be worthwhile to compare presumptive lens tissues under the two different temperatures in terms of expression of developmental genes, particularly those regulating crystallin genes now identified (Kondoh, 1999; Kondoh et al., 2004; Reza and Yasuda, 2004).

\section{Different cell lineages leading to lens development}

The problems raised in relation to lens development become even more intriguing when lens regeneration is considered. It was already known in the 18th century that some animals, even in adult form, can regenerate a damaged lens, particularly newts and other aquatic animals (see Saha, 1991; Okada, 2000). The process of lens regeneration in newts is especially remarkable, since the newly regenerating lens is derived from the dorsal iris which is a derivative of the eye-cup in ontogeny. This way of lens formation is quite distinct from the normal development in which the lens anlage is located in the head ectoderm. Historically, this iris-derived lens regeneration is called "Wolffian lens regeneration" commemorating a discoverer of the phenomenon.

When turning our attention to another very popular amphibian, Xenopus, a so-called model animal in contemporary developmental 


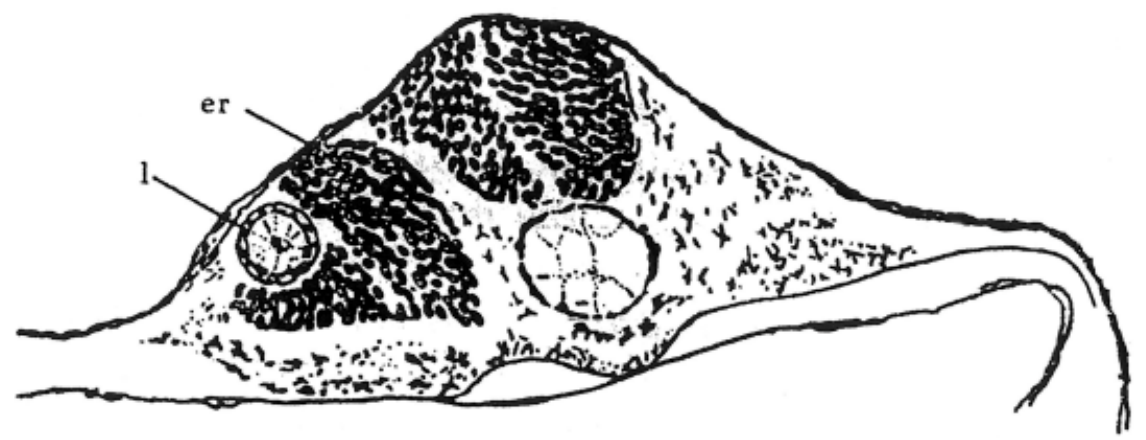

Fig. 2. Lens induction by the optica vesicle transplanted into the ectoderm of a pike embryo (from Filatov, 1935; cited in Dettlaff and Vassetzky, 1997).

biology, lens regeneration occurs from the same source as in ontogeny, i.e. the inner layer of the outer cornea deriving from the head ectoderm. Thus, in contrast to the case of the newt, lens regeneration in Xenopus is essentially a repeat of that which happens during ontogeny, although some differences in the sequence of crystallin gene expression were noted (Mizuno et al., 1999).

Thus, to accomplish the same goal of lens formation, distinct developmental programs are employed among different species, and between ontogeny and regeneration processes even in one animal. Stone (1967) carefully examined different urodele species, 21 species in total, studying the ability and origin of lens regeneration. All species belonging to Triturus (some previously known as Diemitylus or Tarichia, now called Cynops) were able to regenerate the lens from the dorsal iris in the Wolffian fashion, while none of four Ambystoma species showed any ability to regenerate lost lens.

What is the requirement for lens regeneration imposed on the life of a newt? A hint for the answer may lie in its aquatic life. Lens regeneration in the Wolffian fashion is also reported for the Japanese fresh water fish Misgurnus anguillicandatus (Cobitidae) (Sato, 1961). This fish is 7-10 $\mathrm{cm}$ long in adulthood and is offered as a delicacy in gourmet restaurants in the eastern part of Japan. The taxonomic relationship between this fish and newts is distant, but the ecological fact that they both live in freshwater ponds or streams suggests their liaison. T. Sato observed habitation of parasites (perhaps trematodes) in the eyes of Misgurnus fish in his study of lens regeneration, and mentioned the observation briefly in one of his papers (Sato, 1961). G. Eguchi (1980) not only confirmed the

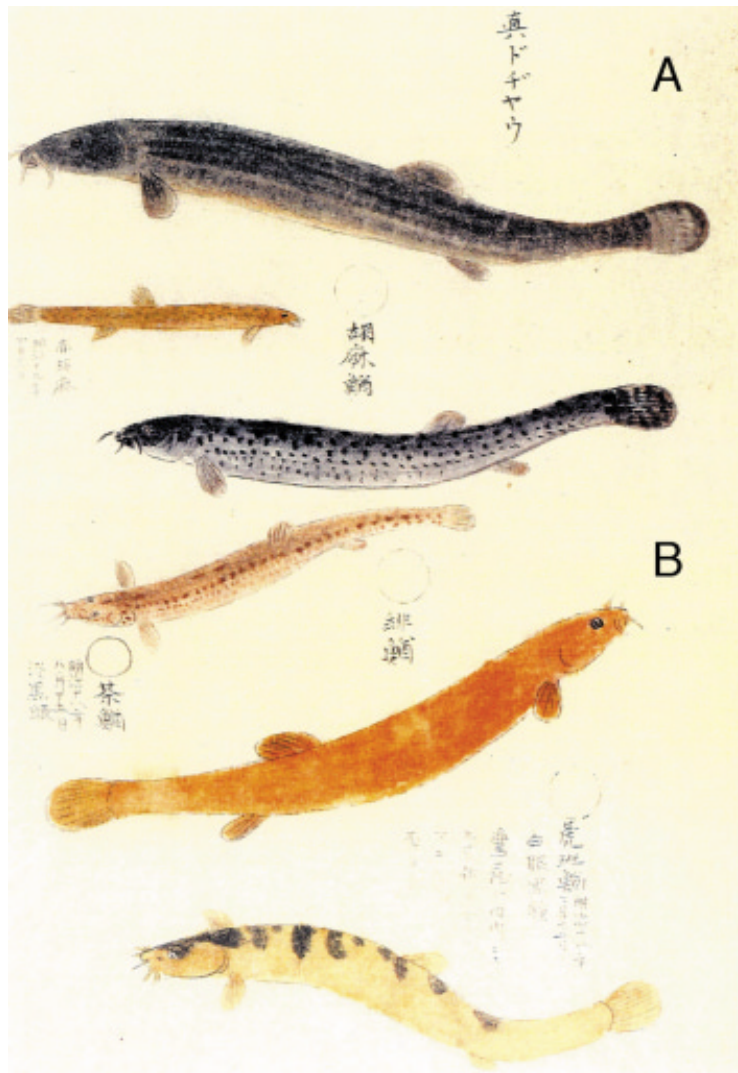

presence of the parasites in the newt eyes but also carefully characterized them (see also Okada, 2000). Because taxonomic identification of this parasite was not complete, G. Eguchi was modest enough not to report this interesting observation internationally. This parasite has a curious and particular appetite for lenses. Accordingly, the lens soon degenerates and disappears from the eye cavity. Lens regeneration in Wolffian fashion from the dorsal iris will then follow, and the poor newt can recover its vision!

Since the last century, an amazing number of examples of tissue regeneration have been reported, each attracting great scientific curiosity. We assume, not necessarily with evidence, that regeneration is beneficial for maintaining a life. Now we have seen a clear case indicating a merit of regeneration to maintain life in the natural environment. Was the lens-regenerating ability of the newts acquired by an adaptive change to resist trematodan attack in the long history of evolution?

Recently, ecological interaction of different species (e.g. trematoda-amphibia) has attracted the interest of developmental biologists, as trematoda-like parasites may cause frequent anomalies (e.g. supernumerary) in developing legs of frogs in certain areas of the world (see Session and Ruth, 1990). Is there any unexpected link between developmental biology and ecology, as implicated by the interaction of the two species affecting morphogenesis in nature? (See S. Gilbert, 2001).

The process of lens regeneration has revealed to us the existence of multiple potential pathways of lens development.

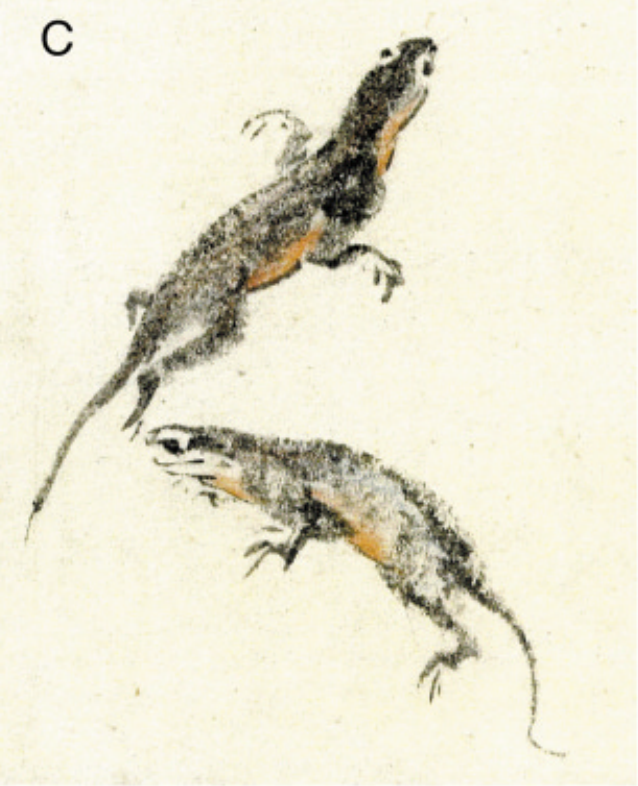

Fig. 3. Champion animals of lens regeneration. Misgurnus anguillicandatis, (A) wild type and (B) a mutant with red color. (C) Cynops pyrrhogaster. Original drawings by unknown Japanese artists from the late 19th century (Dr. Okada's personal colletion). 
Under in vitro culture conditions, it has been demonstrated that almost all non-lenticular ocular tissues, e.g. pigmented retina, neural retina, pineal body etc., switch their characteristics and undergo transdifferentiation into the lens (reviewed in Okada, 1991). This demonstrates a wide distribution of lens-generating potency through the non-lenticular ocular tissues, and that this potency is not limited to amphibians which can regenerate lenses from non-lenticular tissues in vivo. The potential of in vitro transdifferentiation has now been demonstrated in a variety of animals which cannot regenerate a lens in vivo. Even adult human-derived pigmented epithelium of the retina can transdifferentiate into a lens (Tsonis et al., 2001). Therefore, the potential of lens regeneration by transdifferentiation may not be limited to newts, and should not remain as a specific interest of zoology. V. Hamburger mentioned in his monograph entitled "The Heritage of Experimental Embryology" (Hamburger, 1988) the cases of transdifferentiation under in vitro culture conditions and of lens regeneration, and stated "the original idea of double assurance', which seemed to be a teleological fantasy, can claim to be the progenitor of a very productive train of thought which contributed substantially to the elucidation of embryonic induction and related phenomena"(cited from Hamburger 1988, p. 70).

Looking back on the history of developmental biology, we note that lens studies have continued to provide landmarks, ever since the discovery of embryonic tissue induction and regeneration by transdifferentiation. Due to the presence of specific marker proteins of the lens, molecular studies of lens development already started before World War II, although using very primitive biochemical techniques at first (see Twitty, 1955; Okada, 2000). These were indeed forerunners of the more recent molecular developmental studies, many of which are well represented in this Special Issue of The International Journal of Developmental Biology. To conclude, studies of lens development continue to be of tremendous interest, not only from a historical perspective, but also because of their contribution to our better understanding of developmental processes.

\section{References}

DETTLAFF, T. A. and VASSETZKY, S. G. (1997). Experimental embryology in Soviet Russia: The Case of Dimitrii P Filatov (1876-1943). Int J Dev Biol. 41: 781-787.

EGUCHI, G. (1980). Regeneration of lens: A switch in differentiation of tissue cells. (Text in Japanese) (Tokyo: Iwanami).

GILBERT, S. F. (2001). Ecological developmental biology: developmental meets the real world. Dev Biol. 233: 1-12.
HAMBURGER, V. (1988). The heritage of experimental embryology. Hans Spemann and the organizer. (Oxford, New York: Oxford University Press).

$\mathrm{KONDOH}, \mathrm{H}$. (1999). Transcription factors for lens development assessed in vivo. Curr Op Gen Dev. 9: 209-216.

KONDOH, H., UCHIKAWA, M. and KAMACHI, Y. (2004). Interplay of Pax6 and SOX2 in lens development as a paradigm of genetic switch mechanisms for cell differentiation. Int J Dev Biol. this issue.

MIZUNO, N., MOCHII, M., TAKAGI, CH., TAKAHASHI, T. C., EGUCHI, G. and OKADA, T. S. (1998). A critical role for the optic vesicle in lens development; A reinvestigation of free lens formation in Cynops pyrrhogaster. Differentiation. 63: 247-252.

MIZUNO, N., MOCHII, M., YAMAMOTO, T. S., TAKAHASHI, T. C., EGUCHI, G. and OKADA, T.S. (1999). Lens regeneration in Xenopus is not a mere repeat of lens development with respect to crystalline gene expression. Differentiation. 64: 143-149.

OKADA, T. S. (1991). Transdifferentiation-Flexibility in cell differentiation (Oxford: Clarendon Press).

OKADA, T. S. (2000). Lens studies continue to provide landmarks of embryology (developmental biology). J Biosci. 25: 133-141.

REZA, M. and YASUDA, K. (2004). Lens differentiation and crystallin regulation: a chick model. Int. J. Dev. Biol. 48: 805-817.

SAHA, M. (1991). Spemann seen through a lens; in A conceptual history of modern embryology; Developmental biology: A comprehensive synthesis. (ed.) GILBERT, S. F. (New York, London: Plenum Press) 7: 91-108.

SAHA, M. S., SPANN, C. L. and GRAINGER, R.M. (1989). Embryonic lens induction: more than meets of the optic vesicle. Cel/ Differ Develop. 28, 153-171.

SANDER, K. and FAESSLER, P.E. (2001). Introducing the Spemann-Mangold organizer: experiments and insights that generated a key concept in developmental biology. Int. J. Dev. Biol. 45: 1-11.

SATO, T. (1961). Uber die Linsenregeneration bei den Cobitiden Fischen I. Misgurnus anguillicaudatus (CANTOR). Embryologia 6: 251-290.

SERVETNICK, M.D., COOK, T.L.Jr. and GRAINGER, R.M. (1996). Lens induction in axolotls: comparison with inductive signaling mechanisms in Xenopus laevis. Int J Dev Biol. 40:755-761.

SESSIONS, S. K. and RUTH, S. B. (1990). Explanation of naturally occurring supernumerary limbs in amphibians. J Exp Zool. 254: 38-47.

SPEMANN, H. (1901). ‘ber Korrelation in der Entwicklung des Auges. Verh Anat Ges. 15: 61-79.

SPEMANN, H. (1938). Embryonic development and induction (New Heaven: Yale University Press).

STONE, L. S. (1967). An investigation recording all salamanders which can and cannot regenerate a lens from the dorsal iris. J Exp Zool. 164: 87-104.

TSONIS P.A., JANG, W., RIO-TSONIS, K. D. and EGUCHI, G. (2001). A unique aged human retinal pigmented epithelial cell line useful for studying lens differentiation in vitro. Int J Dev Biol. 45: 753-758.

TWITTY, V. (1955). An Analysis of Development. (ed.) WILLIAM, B. H., WEISS, P. A. and HAMBERGER, V. Chapter 2, section VII, pp.402-414. W. B. Sanders Co., Philadelphia and London. 